\title{
Cutis Marmorata Telangiectático Congénito: A Propósito de Cuatro Casos
}

\author{
DANIELA KRÄMER H. ${ }^{1}$, PATRICIA ALFARO C. ${ }^{2}$, PAULA MUÑOZ M. ${ }^{1}$ \\ 1 Dermatólogo. Unidad de Dermatología, Hospital Luis Calvo Mackenna. \\ 2 Médico en etapa de destinación y formación.
}

\begin{abstract}
Congenital Cutis Marmorata Telangiectasia: Four Case Review

Congenital Cutis Marmorata Telangiectasia (CCMT) is a skin vascular malformation of low flow compromising venous and capillary vessels. Of unknown origin, it shows in an estimated $1 / 3000$ births. In over $90 \%$ of cases it can be seen since birth, although it can appear on the first few days after. Clinical manisfestation includes poorly defined reticular erythematopurpureal maculae, frequently associated to areas of segmental, asymetric skin atrophy, most often seen in the lower extremitie. The diagnosis is mostly clinical, important to make as it is associated in $30 \%$ of cases to other congenital abnormalities. No specific treatment is required, with spontaneous resolution. Prognosis is good, depending on the associated malformations. Four patients are described in this paper, as a reminder of the clinical entity.
\end{abstract}

(Key words: Congenital cutis marmorata telangiectasia, vascular malformation, congenital abnormalitie).

Rev Chil Pediatr 2010; 81 (3): 241-245

\section{RESUMEN}

El Cutis Marmorata Telangiectático Congénito (CMTC) es un tipo de malformación vascular cutánea de bajo flujo que compromete vasos capilares y venosos. Su causa es desconocida y tiene una incidencia estimada de 1/3 000 recién nacidos. En más del 90\% de los casos se presenta desde el nacimiento. Sin embargo, también puede aparecer en los primeros días de vida. Clínicamente se observan máculas eritematovioláceas, de aspecto reticulado, de bordes mal definidos, asociado frecuentemente a zonas de atrofia cutánea, de distribución segmentaria, asimétricas. Se ubican de preferencia en las extremidades, siendo las inferiores las más afectadas. El diagnóstico es fundamentalmente clínico y su importancia radica en que aproximadamente el 30\% de los casos se asocia a otras anomalías congénitas, la mayoría de carácter leve. No tiene un tratamiento específico, ya que evoluciona en poco tiempo a la resolución en forma espontánea. El pronóstico es generalmente bueno, aunque depende de las anomalías asociadas. En este artículo se presentan cuatro pacientes cuya historia clínica es compatible con este diagnóstico, para dar a conocer esta entidad clínica a los pediatras.

(Palabras clave: Cutis marmorata telangiectático congénito, anomalía vascular).

Rev Chil Pediatr 2010; 81 (3): 241-245

Trabajo recibido el 03 de noviembre de 2009, devuelto para corregir el 29 de diciembre de 2009, segunda versión el 05 de abril de 2010, aceptado para publicación el 15 de abril de 2010.

Correspondencia a:

Daniela Krämer $\mathrm{H}$.

E-mail: danielakramer.chile@gmail.com 


\section{Introducción}

Las lesiones vasculares de la infancia son anomalías cutáneas que se producen por errores en la vasculogénesis y que dan como resultado diferentes manifestaciones clínicas. Se clasifican según criterios clínicos, radiológicos e histológicos en tres grupos: tumores, malformaciones y dilataciones vasculares.

Las malformaciones vasculares congénitas de la piel son anomalías causadas por una alteración en la morfogénesis de los vasos ocurridos probablemente entre la cuarta y décima semana de vida intrauterina. Se caracterizan por estar presentes desde el nacimiento y crecer en forma proporcional al crecimiento del niño.

Pueden clasificarse según el vaso predominante, afectando a cualquier rama vascular ya sea capilar, arterial, venosa o linfática. También se clasifican según las características del flujo, dividiéndose en lesiones de alto y bajo flujo. Las lesiones de alto flujo son de origen arterial o arteriovenoso, y las de bajo flujo comprometen vasos capilares, venosos y/o linfáticos, observándose combinaciones de los diferentes tipos. Además, clínicamente pueden presentarse como lesiones aisladas o asociadas a otras malformaciones, conformando diferentes síndromes.

El Cutis Marmorata Telangiectático Congénito (CMTC) es un tipo de malformación vascular cutánea de bajo flujo que compromete vasos capilares y venosos. Su causa es desconocida y tiene una incidencia estimada de 1/3000 recién nacidos, con un leve predominio en el sexo femenino. En más del $90 \%$ de los casos se presenta desde el nacimiento. Sin embargo, también puede aparecer en los primeros días de vida ${ }^{1}$.

Un alto porcentaje de casos se asocia a otras anomalías congénitas y en este contexto representa un importante desafío para el profesional, ya que su diagnóstico adecuado y oportuno permitirá orientar adecuadamente el estudio y por otra parte permitirá informar a los padres acerca del pronóstico de la enfermedad.

Presentamos un estudio retrospectivo descriptivo de cuatro casos de CMTC, diagnosticados entre los años 2001 y 2009 en la Unidad de Dermatología del Hospital Luis Calvo Mackenna. El objetivo de este artículo es exponer a la comunidad pediátrica las características clínicas que permiten identificar esta patología.

\section{Casos Clínicos (Tabla 1)}

\section{Caso Clínico $\mathrm{N}^{0} 1$}

Lactante de 3 meses de edad, de sexo masculino, sin antecedentes mórbidos de importancia. Consulta por máculas planas de color violáceo asociadas a atrofia del tejido celular subcutáneo circundante, que se observan desde el nacimiento y comprometen completamente

Tabla 1. Resumen hallazgos clínicos

\begin{tabular}{|lllll|}
\hline Edad de inicio & Sexo & Cuadro clínico & Ubicación & Lesiones asociadas \\
\hline Recién Nacido & Masculino & $\begin{array}{l}\text { Máculas violáceas, con atrofia } \\
\text { en tejido celular subcutáneo } \\
\text { circundante }\end{array}$ & $\begin{array}{l}\text { Extremidad superior derecha, } \\
\text { zona pectoral y deltoídea } \\
\text { derecha, fosa lumbar derecha } \\
\text { hasta la línea media }\end{array}$ & No \\
Recién Nacido & Femenino & $\begin{array}{l}\text { Máculas rosadas hipopigmen- } \\
\text { tadas de aspecto reticulado }\end{array}$ & $\begin{array}{l}\text { Región antero medial del } \\
\text { muslo y pierna derecha }\end{array}$ & No \\
\hline Recién Nacido & Femenino & $\begin{array}{l}\text { Máculas de color rojo inten- } \\
\text { so, de aspecto marmóreo con } \\
\text { atrofia importante }\end{array}$ & $\begin{array}{l}\text { Extremidad superior e inferior } \\
\text { izquierda }\end{array}$ & $\begin{array}{l}\text { Mancha mongólica en hom- } \\
\text { bro derecho; Mancha café } \\
\text { con leche en abdomen }\end{array}$ \\
Recién Nacido & Femenino & $\begin{array}{l}\text { Máculas violáceas de aspecto } \\
\text { reticulado }\end{array}$ & Extremidad inferior izquierda & $\begin{array}{l}\text { Mancha salmón en párpado } \\
\text { superior izquierdo; Mancha } \\
\text { café con leche en pierna } \\
\text { izquierda }\end{array}$ \\
\hline
\end{tabular}


extremidad superior derecha, zona pectoral y deltoídea y fosa lumbar ipsilateral, extendiéndose hasta la línea media, donde bruscamente se interrumpen. Además, presenta una lesión similar en extremidad inferior izquierda, comprometiendo zona del muslo y disminución del perímetro del brazo derecho y del tamaño de la mano derecha. Los padres refieren que las lesiones se han tornado menos notorias con el paso de los días (figura 1).

\section{Caso Clínico $\mathrm{N}^{\circ} 2$}

Lactante de 1 mes de edad, de sexo femenino, sin antecedentes mórbidos de importancia. Desde el nacimiento presenta máculas rosadas e hipo pigmentadas de aspecto reticulado en región antero medial del muslo y pierna derechos, asociado a atrofia leve en algunas zonas de la lesión. El color de la lesión se intensifica con el frío y se hacen más notorias en bipedestación. No presenta asimetría del grosor y largo de las extremidades. La radiografía y ecografía doppler de la extremidad comprometida resultaron normales. Fue dada de alta a los 4 años, con escasas lesiones y con disminución notoria de la coloración de éstas.

\section{Caso Clínico $\mathrm{N}^{o} 3$}

Lactante de 10 meses de edad, de sexo femenino, sin antecedentes mórbidos de importancia. Desde el nacimiento presenta extensas máculas de color rojo intenso de aspecto

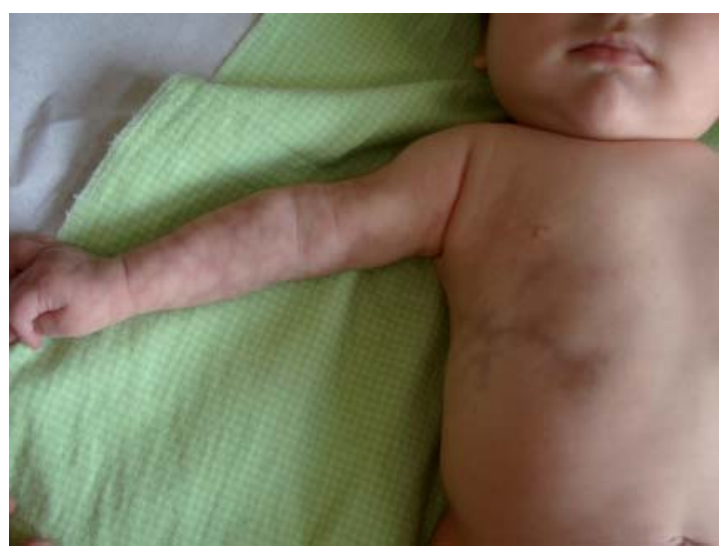

Figura 1. Caso clínico $\mathrm{N}^{\circ} 1$. Mácula color violáceo con atrofia del tejido subcutáneo circundante, en extremidad superior derecha. marmóreo, asociadas a atrofia, ubicadas en extremidad superior e inferior izquierdas, con adelgazamiento importante del grosor del brazo izquierdo. Además, se observan dos manchas mongólicas en el hombro y región lumbar del lado derecho y una mancha café con leche en el abdomen. La radiografía y ecografía doppler de las extremidades comprometidas y la ecografía lumbosacra fueron normales. En controles al año, dos y tres años, se observó atenuación de las lesiones y recuperación de la simetría del grosor de las extremidades, manteniendo sólo pequeñas máculas reticuladas rojizas que desaparecen a la compresión. Fue dada de alta a los 4 años.

\section{Caso Clínico $\mathrm{N}^{\circ} 4$}

Lactante de 2 meses de edad, de sexo femenino, sin antecedentes mórbidos de importancia. Desde el nacimiento presenta máculas violáceas de aspecto reticulado en extremidad inferior izquierda. Presenta además una mácula salmón en el párpado superior izquierdo y una mancha café con leche en la extremidad inferior izquierda. Los padres observan atenuación de las lesiones con el paso de los días (figura 2).

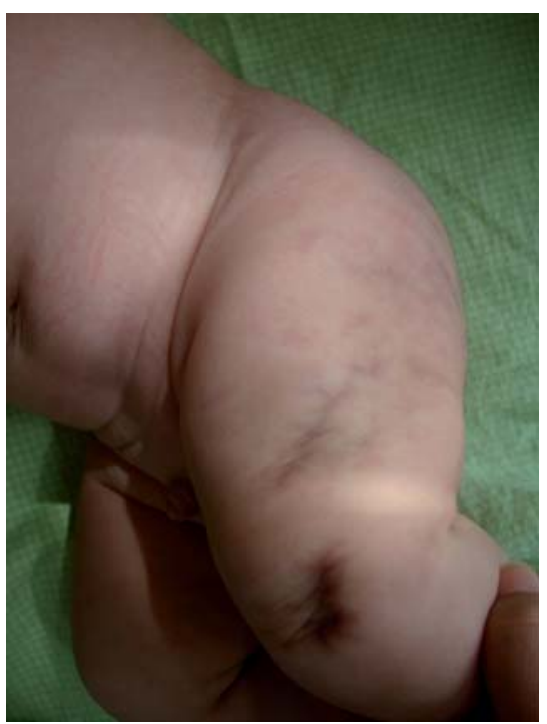

Figura 2. Caso clínico $\mathrm{N}^{\circ}$ 4. Mácula violácea de aspecto reticulado, en extremidad inferior izquierda. 


\section{Cuadro Clínico}

En el CMTC se observan máculas eritematovioláceas, de aspecto reticulado, de bordes mal definidos, asociadas frecuentemente a zonas de atrofia cutánea, de distribución segmentaria, asimétricas. Se ubican de preferencia en las extremidades, siendo las inferiores las más afectadas. También puede observarse compromiso del tronco y la cabeza, y es poco frecuente en mucosas, palmas y plantas ${ }^{2-4}$.

Por otra parte, se observa que el color de las lesiones se intensifica con el frío, el ejercicio y el llanto, y se atenúa con la compresión, mientras que el aumento de la temperatura no produce cambios en la coloración. Sobre éstas lesiones, pueden aparecer otras tales como telangectasias, hiperqueratosis y flebectasia, además de trastornos de la simetría y perímetro de las extremidades comprometidas, sobre todo en la piel de los codos y las rodillas. Una de las principales características de esta patología es que las lesiones cutáneas evolucionan a la resolución en forma espontánea.

\section{Diagnóstico}

El Diagnóstico es fundamentalmente clínico. Los estudios complementarios deben reservarse para los casos en los que los hallazgos clínicos lo ameriten. El estudio histológico no está indicado ya que es inespecífico.

La importancia del diagnostico radica en que aproximadamente el $30 \%$ de los casos se asocia a otras anomalías congénitas, tales como alteraciones músculo esqueléticas, oculares y cutáneas ${ }^{1,5}$. Sin embargo, la mayoría de estas son de carácter leve. La anomalía musculo esquelética más frecuente es el aumento o disminución del perímetro de las extremidades comprometidas, presentándose también fisura palatina, micrognatia, dientes distróficos y macrocefalia. En cuanto a las anomalías cutáneas, las que se observan con mayor frecuencia son el Nevus Flammeus, Aplasia Cutis Congénita, Hemangiomas, Manchas Café Con Leche y Manchas Mongólicas. Por otra parte, el Glaucoma Congénito es la anomalía ocular asociada descrita con mayor frecuencia ${ }^{1,3,4,6}$.

\section{Diagnóstico Diferencial}

El Diagnóstico Diferencial debe incluir otras lesiones que se presentan con aspecto reticulado, tales como el Cutis Marmorata Fisiológico del recién nacido, Nevus Flammeus, lesiones precursoras de hemangiomas de la infancia y Lupus Eritematoso Neonatal ${ }^{1}$.

El Cutis Marmorata Fisiológico del recién nacido se presenta como respuesta a la hipotermia, observándose máculas de aspecto reticulado y de distribución simétrica, principalmente en el tronco y en las extremidades. Además, el color de las lesiones se atenúa con el aumento de la temperatura y no hay atrofia en la piel comprometida, características que lo que lo diferencian del CMTC.

El Nevus Flammeus se presenta como una mácula rojiza, bien delimitada, de ubicación unilateral, cuya coloración no se atenúa con el paso de las semanas, y a diferencia de la atrofia observada en el CMTC, evoluciona con los años a la hipertrofia.

En el Lupus Eritematoso Neonatal se describe un eritema de aspecto reticulado, principalmente de ubicación facial y distribución simétrica, acompañado de telangectasias $\mathrm{y}$ atrofia de la piel circundante.

Por otro lado, en algunas anomalías genéticas tales como Trisomía 21, Síndrome de Cornelia de Lange y Homocistinuria, se observan tenues máculas eritematosas congénitas con patrón reticular.

Es importante mencionar que el CMTC puede asociarse a otras malformaciones congénitas conformando diferentes síndromes. Ejemplos de esto son el Síndrome de Adams Oliver y el Síndrome Macrocefalia- CMTC.

El Síndrome de Adams Oliver (CMTC con Aplasia Cutis) es un síndrome de herencia autosómica recesiva que se manifiesta con gran variabilidad clínica. Sus principales características son la presencia de aplasia cutis del cuero cabelludo y malformaciones de los brazos, piernas y dedos de las manos y pies. El 20\% de los casos presentan Cutis Marmorata Telangiectático, que regresa espontáneamente en los primeros años de vida. El diagnóstico es fundamentalmente clínico, y se confirma con estudio de imágenes que muestran las malformaciones esqueléticas. No tiene tratamiento específico. 
El Síndrome Macrocefalia-CMTC es un cuadro caracterizado por la presencia de macrocefalia y Cutis Marmorata de localización nasal y en el surco de la línea media del labio superior (filtrum), en relación a la ubicación de los mamelones frontonasal y nasal medial durante el desarrollo embrionario de la cara. Estos signos pueden asociarse a macrosomía fetal, asimetría de las extremidades inferiores, sindactilia del segundo, tercero y/o cuarto ortejo, retraso del desarrollo psicomotor leve a moderado y anomalías del tejido conectivo, tales como hiperlaxitud cutánea e hiperextensibilidad articular. Al igual que el Síndrome de Adams Oliver, tampoco tiene tratamiento específico.

\section{Tratamiento y Pronóstico}

No existe un tratamiento específico para esta patología, ya que las lesiones evolucionan espontáneamente a la resolución en un $90 \%$ de los casos durante los dos o tres primeros años de vida ${ }^{1}$. Por lo tanto, el pronóstico es generalmente bueno, aunque depende de las anomalías asociadas.

Se ha planteado que las lesiones cutáneas persistentes podrían tratarse con láser de colorante pulsado, aunque la respuesta es variable y aún no ha sido analizada en el largo plazo ${ }^{1,7,8}$.

\section{Comentarios}

El CMTC es una enfermedad benigna, poco frecuente $\mathrm{y}$, en general, de buen pronóstico; su diagnóstico es eminentemente clínico y a menudo es suficiente una historia clínica completa y un exhaustivo examen físico para descartar las posibles anomalías asociadas.

Queremos destacar la importancia de tener presente este diagnóstico cuando nos enfrentamos a un recién nacido con lesiones reticuladas eritematovioláceas, principalmente para realizar un buen diagnóstico diferencial y orientar el estudio complementario en caso de ser necesario. Un buen diagnóstico inicial es importante para informar adecuadamente a los padres acerca de la naturaleza y evolución de estas lesiones.

\section{Referencias}

1.- Del Boz González J, Serrano Martín M, Vera Casaño A: Cutis Marmorata Telangiectasica Congénita: Review of 33 cases. An Pediatría (Barc) 2008; 69: 557-64.

2.- Pehr K, Moroz B: Cutis Marmorata Telangiectatica Congenita: Long-term follow-up, review of the literature and report of a case in conjunction with congenital hypothyroidism. Pediatr Dermatol 1993; 10: 6-11.

3.- South DA, Jacobs AH: Cutis Marmorata Telangiectasica Congénita (congenital generalizad phlebectasia). J Pediatr 1978; 93: 944-9.

4.- Nagore A, Torrelo A, Zambrano A: Cutis Marmorata Telangiectasica Congénita. Revisión de 28 casos. Actas Dermosifiliogr 1999; 90: 433-8.

5.- Hinek A, Jain S, Taylor G, Nykanen D, Chitayat D: High copper levels and increased elastolysis in a patient with Cutis Marmorata Telangiectasica Congenital. Am J Med Genet A 2008; (19): 2520-7.

6.- Lee S, Lee JB, Kim JH, Kim KY, Lee SH: Cutis Marmorata Telangiectatica with multiple congenital anomalies (Van Lohuizen's syndrome). Dermatol 1981; 163: 40812.

7.- Baniandrés Rodríguez O, Boixeda de Miquel P, Nieto Perea O, Pérez Rodríguez A, Belmar Flores P, Arrazola Mallona JM: Cutis Marmorata Telangiectasica Congénita. Actas Dermosifiliogr 2003; 94: 31-110.

8.- Llorca M, González de Dios J, Navarro Belmonte MR: Tratamiento con láser de las anomalías vasculares cutáneas en la infancia: análisis prospectivo de nuestra experiencia en 95 niños. An Pediatr 2000; 52: 6-14.

9.- Amitai DB, Fichman S, Merlob P, Morad Y, Lapidoth M, Metzker A: Cutis Marmorata Telangiectasica Congénita Clinical findings in 85 patients. Pediatr Dermatol 2000; 17: 100-4.

10.- Lobo-Mueller E, Amaral JG, Babyn PS, Wang Q, John P: Complex combined vascular malformation syndromes affecting the extremities in children. Semin Musculoskelet Radiol 2009; 13 (3): 255-76.

11.- Bormann G, Wohlrab J, Fischer M, Marsch WC: Cutis Marmorata Telangiectatica Congenital: Laser doppler fluxmetry evidence for a functional nervous defect. Pediatr Dermatol 2001; 18: 110-3.

12.- Kienast AK, Hoeger PH: Cutis Marmorata Telangiectasica Congenita: a prospective study of 27 cases and review of the literature with proposal of diagnostic criteria. Clin Exp Dermatol 2009; 34 (3): 319-23. 